RUNNING HEAD: The social presence effect of eye tracking

\title{
Wearable computing: Will it make people prosocial?
}

To appear in British Journal of Psychology, 2014

\author{
Eleni Nasiopoulos ${ }^{1}$, Evan F. Risko ${ }^{2}$, Tom Foulsham ${ }^{3}$, and Alan Kingstone ${ }^{1}$ \\ ${ }^{1}$ University of British Columbia, ${ }^{2}$ University of Waterloo, \& ${ }^{3}$ University of Essex
}

Corresponding Author: Eleni Nasiopoulos, Department of Psychology, University of BC, Vancouver BC, V6T 1Z4. E-mail: enasiopoulos@psych.ubc.ca. Telephone: + (604)-822-0069 


\begin{abstract}
We recently reported that people who wear an eye tracker modify their natural looking behaviour in a prosocial manner. This change in looking behaviour represents a potential concern for researchers who wish to use eye trackers to understand the functioning of human attention. On the other hand, it may offer a real boon to manufacturers and consumers of wearable computing (e.g., Google Glass), for if wearable computing causes people to behave in a prosocial manner, then the public's fear that people with wearable computing will invade their privacy is unfounded. Critically, both of these divergent implications are grounded on the assumption that the prosocial behavioural effect of wearing an eye tracker is sustained for a prolonged period of time. Our study reveals that on the very first wearing of an eye tracker, and in less than 10 minutes, the prosocial effect of an eye tracker is abolished, but by drawing attention back to the eye tracker the implied presence effect is easily reactivated. This suggests that eye trackers induce a transient social presence effect which is rendered dormant when attention is shifted away from the source of implied presence. This is good news for researchers who use eye trackers to measure attention and behaviour; and could be bad news for advocates of wearable computing in everyday life.
\end{abstract}

Words:217

Keywords: Social Presence, Wearable Computing, Attention, Eye Movements 
Social presence effect of wearable computing 2

\section{Introduction}

It is established that the presence of others elevates conformance to social norms, which reflects one's need to attain approval or avoid the disapproval of others (Guerin, 1986). In the physical presence of others, people will reduce levels of food intake (Herman, Roth \& Polivy, 2003) and alter the way they express emotions (Buck, Losow, Murphy \& Costanzo, 1992). Social presence effects, however, extend beyond the physical presence of others. For example, security cameras, which imply the presence of others, increase prosocial behaviours, with individuals being more likely to provide help to others in a public setting (Van Rompay, Vonk \& Fransen, 2009).

The influence of an implied social presence has recently been extended to the domain of visual attention vis-a-vis eye tracking (Risko \& Kingstone, 2011). One of the working assumptions underlying eye tracking research is that typical looking behaviour is unaffected by wearing the eye tracker and the knowledge that one's eyes are being monitored. It was surprising then when Risko and Kingstone (2011) discovered that looking behaviour is affected by wearing an eye tracker. When provided with the opportunity to look at a sexy swimsuit calendar mounted on a wall, the vast majority of participants chose not to do so if their eyes were being monitored. However, if participants believed that the eye tracker was turned off, or if they were not wearing an eye tracker at all, then they looked at the swimsuit calendar. In short, participants change their looking behaviour when they know or believe that their eye movements are being recorded by an eye tracker. The eyetracker functions as an implied social presence that leads individuals to adjust their looking behaviour in a prosocial manner.

This finding has potentially serious, negative implications for researchers that use eye trackers to understand the mechanisms of human attention and cognition. In a nutshell, the 
concern is that if wearing an eye tracker changes behaviour to the extent that people actively avoid looking at things that they would look at in a more natural environment, then researchers run the risk of drawing conclusions regarding human attention and interest that are precisely the opposite to reality. For instance, if Risko and Kingstone (2011) had only run the standard eye tracking condition then the data would have supported the conclusion that when left alone in a room the vast majority of people do not look at sexy images of women. In fact, the truth is anything but that.

It is interesting to note that what might be bad news for basic eye movement researchers could be good news for people who wear or manufacture wearable computing. It is no exaggeration to say that there are profound concerns that wearable computing will record people in places where they expect personal privacy to be respected (e.g., in a changing room, in one's personal office space at work, or when sharing a private moment at a restaurant). However, to the extent that wearable computing might reveal where one's attention is directed (e.g., Google Glass recording one's head turn to look at an attractive individual) the data from Risko and Kingstone (2011) suggest that people will adopt prosocial looking behaviours and avoid acts that would present them in a negative light.

Both the potentially bad news for eye movement researchers and good news for users of wearable computing turns on the assumption that the prosocial effect of the technology is sustained for a prolonged period of time. An alternative is that the social presence effect triggered by an eye tracker is transient, and quickly dissipates. Addressing this issue is the focus of the present investigation. 


\section{Present Investigation}

Whether eye tracker induced social presence exerts a sustained strong effect or a transient weak effect touches on the methodological and theoretical implications of the phenomenon. Methodologically, determining the relative strength of the effect can inform research decisions. A relatively sustained presence effect might require eye tracking researchers to include control conditions that monitor eye gaze surreptitiously, whereas a transient presence effect might require a far less extreme solution. Theoretically, determining the relative strength of the eye tracker induced social presence effect will lay the foundation for comparison to social presence effects induced by other means ranging from surveillance cameras to the very belief in the presence of supernatural watchers (Shariff \& Norenzayan, 2007), and it will also provide important clues as to the mechanisms underlying these effects (e.g., Crosby, Monin \& Richardson, 2008; Dale \& Vinson, 2013).

To our knowledge, there is no experimental work systematically investigating the extent to which a given social presence effect, once induced, can be reduced or even eliminated, and certainly no work on this issue in the context of eye tracker induced social presence effects. Legitimate cases can be made for both the sustained and transient presence hypotheses. In favour of the sustained hypothesis there exists solid and converging evidence that the eyes represent a critical social stimulus (Birmingham \& Kingstone, 2009; Risko et al., 2012). Thus, prolonged monitoring of the eyes could be expected to induce a durable form of social presence. In a similar vein, social presence effects in general might be expected to be relatively durable given the amount of time individuals spend in social settings. These data converge on the prediction that when an eye tracker is present, prosocial looking behaviour will be the norm. Only knowledge that one's eyes are not being watched (e.g., removal of the eye tracker) will 
yield looking behaviour that is deviant from the prosocial norm (e.g., people will look at the sexy calendar). We call this "natural looking behaviour" in that participants are doing what they would naturally do when they do not feel like their eyes are being watched. On the other hand, eye tracking is both novel to most individuals and designed to be non-invasive. Both of these features could lead individuals wearing an eye tracker, with time or distraction, to revert to a state akin to when their eyes were not being monitored, thus leading them to a more natural pattern of looking. In this case, the eye tracker induced social presence effect would represent a transient social presence effect in the sense that its influence diminishes despite the continued presence of the eye tracker.

To test between the sustained and transient presence hypotheses, we modified the paradigm developed by Risko and Kingstone (2011). The modified task involved three phases. In the first phase participants took part in an easy and engaging task outside the laboratory, searching a building for coloured items mounted on the walls (phase 1). The second and third phases constitute a direct replication of Risko and Kingstone. Participants were placed in a room with a sexy swimsuit calendar (with only female models depicted) on the wall and were asked to complete a simple computer based task (phase 2) followed by one minute of "free time" while they waited to be retrieved by the experimenter (phase 3). The critical manipulation involved if and when an eye tracker was worn.

There were four groups. In the no eye tracker group, the participant did not wear an eye tracker during any phase of the experiment. This represents the natural looking group and we expected participants would look at the calendar during phase 3. In the standard eye tracker group, participants wore an eye tracker during the second and third phases of the experiment. This replicates Risko and Kingstone, and we expected participants would avoid looking at the 
calendar. In the all phases eye tracker group participants wore the eye tracker during phases 1 through 3. Thus, participants in the all phases group had more time to wear the eye tracker and a more varied set of experiences (including experience outside the laboratory) wearing the eye tracker both of which could lead to individuals (if possible) behaving as if the eye tracker was no longer present. According to the sustained presence hypothesis, the all phases group should behave like the standard group, whereas according to the transient presence hypothesis the all phases group should behave like the no eye tracker group. For our final group, the recalibration group, participants wore the eye tracker for all 3 phases, but we added a second calibration between phase 1 and 2 . If the social presence effect is found to be transient, then the recalibration group will reveal whether the device has been habituated to or not. If the participant has habituated to the device - defined as a decrease in responsiveness upon repeated (continuous) exposure to the eye tracker - then drawing participants' attention back to the eye tracker should have little effect (e.g., they have ceased to care about it). On the other hand, if the transient effect reflects participants simply shifting attention away from the tracker, then the social presence effect should be easily reactivated by a simple reminder that their eye movements are being recorded by the tracker.

\section{Method}

\section{Participants}

59 females and 23 males, ranging in age from 18 to 51 years, volunteered. Participation was contingent on normal or corrected vision (contacts), and resulted in $\$ 5$ payment or course credit. Participants provided consent, and although deception was necessary for the study, they were debriefed and the true purpose of the study was explained when they completed the 
experiment. Participants had the option to withdraw their data (the hidden video recording and all other data) if they chose to do so.

\section{Apparatus \& Stimuli}

An ASL MobileEye was used for eye tracking, which consists of a head mounted system with the glasses connected to a digital video recorder that records information about the eye and the scene. A pinhole camera (indiscernible from the distance participants were seated at) was hidden behind the provocative stimulus at a $90^{\circ}$ angle to the right of where participants sat for the computer task.

\section{Procedure}

Participants read and signed a consent form before the study. In the all phases eye tracker group and the recalibration group participants were fitted with the eye tracker and a calibration was conducted in the laboratory. Participants were told that they would have their eyes tracked and were aware of the moment that recording began. Participants then commenced the 'counting colours task' which involved walking freely on a specific level of a campus building and searching for and counting coloured squares that were posted on the walls in various locations. These squares were either blue or green and participants were given 2.5 minutes to count squares belonging to one of the two colours and another 2.5 minutes counting the other coloured squares. The experimenter accompanied them into the halls while keeping time. Participants were told that they would be reporting how many squares they remembered counting for each colour. In the standard eye tracker group and the no eye tracker group, participants completed the same 5-minute task without the eye tracker. Upon completion of the counting colours task, participants were directed to the laboratory, where they wrote down the 
number of squares they remembered counting. All participants found this task simple and straightforward and performed it nearly flawlessly.

After the counting colour task participants in the recalibration group did a second recalibration of the eye tracker, and the participants in the standard eye tracker group were fitted with the mobile eye tracking device and the calibration was conducted. All participants then proceeded to perform a computer-based task. This filler task was an exact replication of the Risko and Kingstone (2011) original task, where participants were asked to complete a 6-minute cueing experiment (Frischen, Bayliss \& Tipper, 2007) alone in a room on a computer. In this room the provocative stimulus was $90^{\circ}$ to the right of the participant. All other contents of the room were held constant across conditions, and the room resembled a typical graduate student office setting with two desks, textbooks, a desktop computer and filing cabinets. Within the swimsuit calendar, a wireless pinhole camera was hidden in order to record participant's looking behaviour. Participants were given instructions for the cueing task and told to wait for the experimenter when they had finished the task. The experimenter was able to observe participants through the hidden camera feed, and once the sham cueing experiment was over, participants were left alone in the room for one minute, while their gaze behaviour towards the calendar was recorded. Once this critical minute had elapsed, the experimenter returned, removed the tracker (if worn), and debriefed the participants.

\section{Results}

The key one minute video clips were coded for looking behaviour. Two raters, naïve to the conditions of the clips, coded for direct looks towards the calendar. Agreement on whether participants looked at the calendar was perfect between the raters, $\mathrm{K}=1.0, \mathrm{p}<.001$, leading to no 
loss of participant data. The total sample size consisted of 82 participants. An analysis of gender showed no gender effects on looking behaviour.

Chi squared analysis with one degree of freedom $(d f=1)$ was used to compare the frequency of people in each group who looked toward the calendar. As in Risko and Kingstone (2011) participants were more likely to look at the provocative stimulus in the no eye tracker group (85\%) than in the standard eye tracking group (45\%). This difference was significant, $\mathrm{X}^{2}=7.29, \mathrm{p}=.007$. Critically, participants in the all phases eye tracker group were as likely to look at the provocative stimulus $(80 \%)$ as participants in the no eye tracker group $\mathrm{X}^{2}=0.12$, $\mathrm{p}=.732$, and were significantly more likely to look at the provocative stimulus than participants in the standard eye tracking group, $\mathrm{X}^{2}=5.94, \mathrm{p}=.015$. This shows that the effect of wearing an eye tracker is transient. In light of this finding the results for the recalibration group are especially informative. Our data reveal that these participants, who wore an eye tracker throughout all phases of the study, but received a recalibration condition just before the computer task, reverted back to a performance level that is identical to the standard eye tracking group (45\%), $\mathrm{X}^{2}=0.00, \mathrm{p}=.976$. Accordingly, participants in the recalibration group were less likely to look at the calendar than participants in the all phases group and the no eye tracker group, $X^{2}=5.30, p=.02$, and $X^{2}=7.136, p=.008$, respectively.

\section{Insert Figure 1}

\section{Discussion}

The results are clear. Replicating Risko and Kingstone (2011) participants tend to look at the calendar when they believe that their eyes are not being monitored (no eye tracker group), and they avoid looking at the calendar when they know that their eyes are being monitored 
(standard eye tracker group). One new finding is that participants who wear an eye tracker during an initial colour counting task (all phases group) look at the calendar in a manner similar to those that never wear an eye tracker. These data are consistent with the eye tracker inducing a transient social presence effect in the sense that its influence dissipates rapidly without removing the inducing stimulus. This can be contrasted with what we termed a sustainable presence effect wherein the behavioural influence of social presence (i.e., the inducing stimulus) remains as long as the inducer is present. Furthermore, results from the recalibration group demonstrate that a simple reminder of the eye tracker (i.e., a recalibration) is sufficient to re-induce the prosocial looking behaviour. The theoretical and methodological implications of these results are discussed below.

In the present study taking part in an additional preliminary colour counting task while wearing the eye tracker led to the elimination of the eye tracker induced social presence effect. The additional task was designed to provide participants with more time wearing the eye tracker, the opportunity to take part in an engaging task while wearing the eye tracker, and time wearing the eye tracker outside of the "socially charged" context created by the presence of the provocative stimulus. The ease with which the eye tracker induced social presence effect was eliminated comes as a surprise. We and others have demonstrated that the eyes are a profoundly important social stimulus (Birmingham \& Kingstone, 2009; Baron-Cohen et al., 1997; Emery, 2000; Kobayashi \& Kohshima, 1997; Risko et al., 2012) and thus monitoring an individual's eyes could be thought of as a rather invasive form of monitoring. In addition, socially appropriate looking patterns are likely highly practiced, nevertheless, the fleeting nature of the eye tracker induced social presence effect would seem to suggest that either (a) socially appropriate looking patterns are themselves difficult to monitor and maintain or (b) the eye 
tracker's activation of these mechanisms is significant but vulnerable to habituation as a user becomes accustomed to the device.

Socially appropriate looking patterns (or at least the mechanisms behind them) likely evolved in the context of interacting with real social agents. Eye trackers of course are not real social agents. Rather, eye trackers (and other forms of implied social presence; e.g., video cameras) indirectly represent other social agents and this fact might be responsible for the fragile nature of the eye tracker induced social presence effect. Convergent with our findings that the effect is transient is a meta-analysis by Sparks and Barclay (2013) who report that exposure to eye-like images will increase cooperative behaviour (e.g., Ernest-Jones et al., 2011), but only when exposure is for a short period of time (e.g., less than several minutes). Collectively these data would suggest that the mechanisms underlying implied social presence effects are vulnerable to habituation, and the power of the eye tracker to induce a social presence effect is quickly drained.

An alternative explanation however is that the social presence effects are transient but the power of the eye tracker to induce a social presence effect is only temporarily inactive. Social presence effects are often attributed to attention shifting from an external to an internal focus (Carver, 1979; Carver \& Scheier, 1981; Duval \& Wicklund, 1972; Van Rompay et al., 2009) where this internal focus leads to impression management. In this theoretical context, the additional time and engaging task could be seen as engendering a shift back to an external focus. If this is the case drawing attention back to the tracker, for instance by doing a simple recalibration, should be sufficient to retrigger the social presence effect.

The data from the recalibration condition were unequivocal on this issue. When participants received a recalibration of the eye tracker they reverted to a performance level that 
was identical to the standard eye tracker condition. In other words, the social presence of the eye tracker was reinstituted and participants again demonstrated a strong tendency to avoid looking at the sexy calendar. Collectively, our data then suggest that the transient social presence effect reflects a shift in attention away from the eye tracker rather than habituation to it.

Our finding that the eye tracker induced social presence effect is transient but sensitive to reactivation has important methodological implications. The observation that looking behaviour can change when individuals know their eyes are being monitored presents a challenge to users of that technology insofar as the goal is to understand how individuals examine the world naturally. The present results suggest that it is possible to address this challenge without having to resort to modes of eye tracking in which the participant is unaware. In other words, with a nominal adjustment in one's research methodology individuals who are wearing an eye tracker will display eye movements that closely approximate their natural looking behaviour despite the fact that those eye movements may be socially charged. However, it is critical that researchers avoid doing anything to draw the attention of the participants to the fact that their eyes are being tracked. This demonstration has implications beyond eye tracking in that individuals will likely exhibit similar behaviour with other monitoring technologies. For example, in clinical ambulatory assessment, the present results suggest that, provided participants are given some time with wearable monitoring devices, they may behave naturally (reducing "reactivity"; Trull \& Ebner-Priemer, 2013).

It is important to note the social implications of such findings as well. With the surge of wearable technologies that are soon to become mainstream, people will have the ability to record what they see and upload such information online, onto social networks and other public forums. Such sensitive information being freely shared and captured clearly raises concerns about 
privacy and social tensions arising from the use of such technology, especially with regards to those who are subjected to being recorded (Baker, Schweitzer \& Risko, 2013). Evidence from our investigation suggests that the wearer will likely adapt quickly to the idea that what they see will be recorded and shared and fail to self-monitor in a prosocial manner, but this issue could potentially be mitigated by reminding the wearer that their looking behaviour is being documented. While this may be bad news for users of wearable computing, our data are potentially good news for eye tracking researchers. People quickly adjust to wearing an eye tracker and look at items in their environment as they would when not wearing an eye tracker. However, our data suggest that this is not due to people habituating to wearing the eye tracker, insofar as its social presence effects are diminished and inert. Rather the transient social presence effect of the eye tracker is merely dormant and it can be reactivated to its initial force by just drawing attention to the tracker. 


\section{References}

Baker, D. A., Schweitzer, N. J., \& Risko, E. F. (2013). Perceived Access to Self-relevant Information Mediates Judgments of Privacy Violations in Neuromonitoring and Other Monitoring Technologies. Neuroethics, 1-8. doi:10.1080/15265160590960924

Baron-Cohen, S., Wheelwright, S. \& Jolliffe, T. (1997). Is there a "language of the eyes"? Evidence from normal adults, and adults with autism or asperger syndrome. Visual Cognition, 4, 311-331. doi:10.1080/713756761

Birmingham E., Kingstone A. (2009). Human social attention: A new look at past, present, and future investigations. Annals of The New York Academy of Sciences, 1156, 118-140. doi: 10.1111/j.1749-6632.2009.04468.x

Buck, R., Losow, J. I., Murphy, M. M., \& Costanzo, P. (1992). Social facilitation and inhibition of emotional expression and communication. Journal of Personality and Social Psychology, 63, 962-968. doi: 10.1037/0022-3514.63.6.962

Carver, C.S. \& Scheier, M.F. (1981). The self-attention-induced feedback loop and social facilitation. Journal of Experimental Social Psychology, 17, 545-568. http://dx.doi.org/10.1016 /00221031(81)90039-1

Carver, C.S. (1979). A cybernetic model of self-attention processes. Journal of Personality and Social Psychology, 37, 1251-1281. 
Crosby, J. R., Monin, B., \& Richardson, D. (2008). Where do we look during potentially offensive behavior? Psychological Science, 19, 226-228. doi:10.1111/j.1467-9280.2008.02072.x

Dale, R. \& Vinson, D. W. (2013). The observer's observer's paradox. Journal of Experimental \& Theoretical Artificial Intelligence, 25, 303-322. doi:10.1080/0952813X.2013.782987

Duval, S. \& Wicklund, R.A. (1972). A theory of objective self awareness. New York: Academic Press.

Emery, N.J. (2000). The eyes have it: The neuroethology, function and evaluation of social gaze. Neuroscience and Biobehavioral Reviews, 24, 581-604. http://dx.doi.org/10.1016/S01497634(00)00025-7

Ernest-Jones, M., Nettle, D. \& Bateson, M. (2011). Effects of images on everyday cooperative behavior: A field experiment. Evolution and Human Behavior, 32, 172-178. http://dx.doi.org/10.1016/j.evolhumbehav.2010.10.006

Frischen, A., Bayliss, A.P. \& Tipper, S. P. (2007). Gaze cueing of attention: Visual attention, social cognition, and individual differences. Psychological Bulletin, 133, 694-724. doi: 10.1037/0033-2909.133.4.694

Guerin, B. (1986). Mere presence effects in humans: A review. Journal of Experimental Social Psychology 22, 38-77. http://dx.doi.org/10.1016/0022-1031(86)90040-5 
Herman, P.C., Roth, D.A. \& Polivy, J. (2003). Effects of the presence of others on food intake: A normative interpretation. Psychological Bulleting, 129, 873-886. doi: 10.1037/00332909.129.6.873

Kobayashi, H. \& Kohshima, S. (1997). Unique morphology of the human eye. Nature, $387,767-768$.

Risko, E.F., Laidlaw, K.E.W., Freeth, M., Foulsham, T. \& Kingstone, A. (2012). Social attention with real versus reel stimuli: Toward an empirical approach to concerns about ecological validity. Frontiers in Human Neuroscience, 6, 143. doi: 10.3389/fnhum.2012.00143

Risko, E.F. \& Kingstone, A. (2011). Eyes wide shut: Implied social presence, eye tracking and attention . Attention, Perception \& Psychophysics, 73, 291-296. doi: 10.3758/s13414010-0042-1

Shariff, A.F. \& Norenzayan, A. (2007). God is watching you: Priming God concepts increases prosocial behavior in an anonymous economic game. Psychological Science, 18, 803809. doi: $10.1111 / \mathrm{j} .1467-9280.2007 .01983 . \mathrm{x}$

Sparks, A., \& Barclay, P. (2013). Eye images increase generosity, but not for long: the limited effect of a false cue. Evolution and Human Behavior, 34, 317 - 322. http://dx.doi.org/ 10.1016/j.evolhumbehav.2013.05.001 
Trull, T. J., \& Ebner-Priemer, U. (2013). Ambulatory assessment. Annual review of clinical psychology, 9, 151-176. doi: 10.1146/annurev-clinpsy-050212-185510

Van Rompay, T. J. L., Vonk, D. J., \& Fransen, M. L. (2009). The eye of the camera: Effects of security cameras on prosocial behavior. Environment and Behavior, 41, 60-74. doi: $10.1177 / 0013916507309996$ 


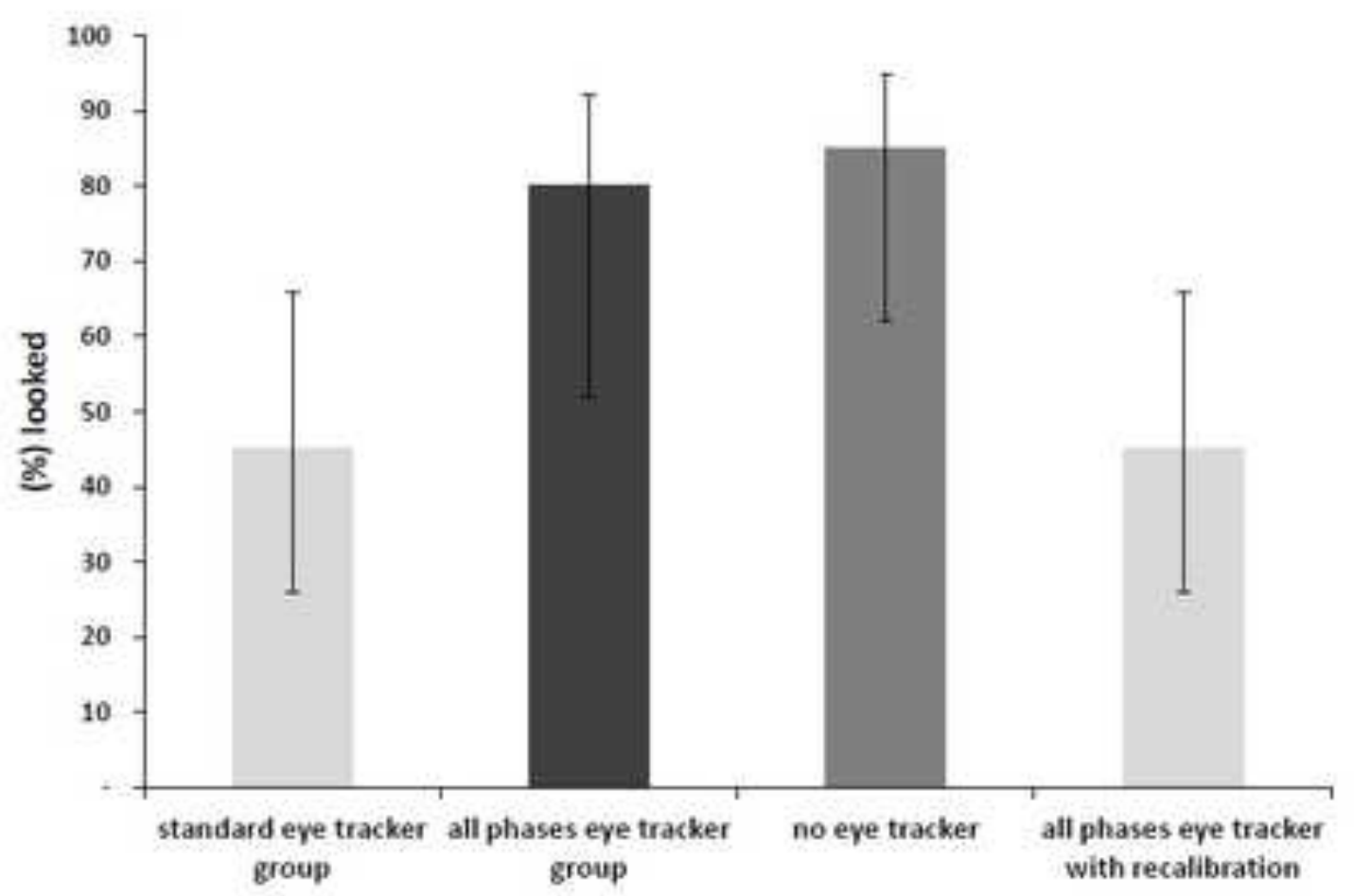

Figure 1. Percentage of participants who looked at the provocative stimulus as a function of group. Error bars indicate the lower and upper limits of the $95 \%$ Confidence Interval for a proportion. 